\title{
Full-Wave Equivalent Network Representation for Multiple Arbitrarily Shaped Posts in $H$-Plane Waveguide
}

\author{
Alejandro Valero, Member, IEEE, and Miguel Ferrando, Member, IEEE
}

\begin{abstract}
This paper presents a method for the analysis of arbitrary shape and composition posts in waveguide. The method segments the problem into regions that are characterized by their generalized admittance matrices. Both an analytical formulation and a boundary integral formulation based on homogeneous wave equation solutions are used in this characterization. Our method is especially fast and suitable for real time computer-aided design tools because only the changing parameters would have to be computed in a design process. This method also makes a simple combination of different numerical techniques possible.
\end{abstract}

Index Terms-Generalized admittance matrix, integral equations, waveguides.

\section{INTRODUCTION}

$\mathbf{T}$ HE rigorous study of arbitrary obstacles such as posts or metallic strips in guide is of constant interest in the design of filters. The initial work of Marcuvitz [1] dealt with small radii and centered posts. Literature since then has improved the description of propagation in guide and, thus, the analysis of more complex and interesting problems has been possible. Nielsen [2] introduced a theory based on modal expansions and pointmatching that has made possible the analysis of posts of greater dimensions. Araneta et al. [3] later introduced a higher order variational method for centrally placed dielectric post of circular cross section. Leviatan and Sheaffer [4] then proposed an integral formulation applicable to any two-dimensional geometry. The finite- and boundary-element methods have also been applied in the works of Ise and Koshiba [5], Hsu and Auda [6], and Wu et al. [7]. Recently, Abdulnour and Marchildon [8] have proposed a method that combines the precision of the modal analysis with the versatility of the boundary-element method.

For the above-mentioned methods, the region that contains the post is analyzed together with the post itself. This implies that in the event of any modification in form, position, or electrical characteristics of this obstacle, the problem would need to be computed from the beginning, thus delaying the design process. The method that we propose segments the problem in regions. Each region is analyzed independently of each other as a boundary-value problem and then each one is characterized by a generalized admittance matrix (GAM).

Manuscript received July 13, 1998.

The authors are with the Departmento de Comunicaciones, Universidad Politécnica de Valencia, 46022 Valencia, Spain (e-mail: avalero@dcom. upv.es).

Publisher Item Identifier S 0018-9480(99)07708-X.

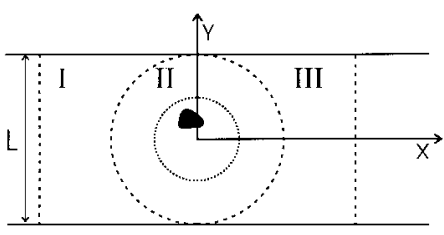

Fig. 1. Description of the problem.

Although GAM formulations have been used extensively for years [9], to the best of our knowledge, a general unified approach considering arbitrary obstacles from this point-ofview is not available. This approach has a double advantage. First, it is not only fast, but also very accurate, as will be shown below. Second, one can use the technique that is best suited to the characteristics of the post.

The problem to be solved is shown in Fig. 1. In the interior of the rectangular waveguide there is a post or group of posts generally not centered and with arbitrary cross section and composition. The most convenient segmentation is the one that establishes a circular boundary around the post so that the scattered field can be formulated in terms of cylindrical waves. On the other hand, the propagation of these waves in the waveguide should be formulated more adequately by means of the solution modes inside it. Fig. 1 shows the applied segmentation. Regions I and III accomplish the transformation from cylindrical to plane waves.

In Section II, the integral formulation that provides the GAM for regions I and III is described. In Section III, an analytical formulation for the central region is described. In Section IV, some discussions are made and some results are shown. Section V then presents conclusions.

\section{INTEGRAL FORMULATION FOR THE ADMITTANCE OF REGIONS I AND III}

The boundary integral equation based on the wave function proposed by Kishi and Okoshi [10] is a valuable tool in the full-wave description of a homogeneous region by means of a GAM. However the formulation originally proposed in [10] has some limitations that should be considered in order to be able to deal with a much wider range of problems than those studied by Kishi and Okoshi. These limitations have to do with the set of solutions of the wave equation and the expansion functions used on the boundary. 


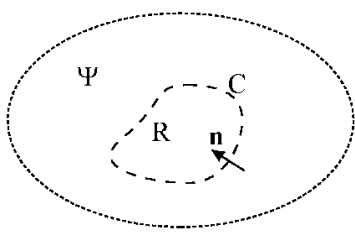

Fig. 2. Closed boundary in a region where the wave function is known.

Let us consider an arbitrary homogeneous region where the wave function $\psi$ is a known solution. Consider also that the problem is two-dimensional and $\mathrm{TM}_{z}$, and take an enclosure $R$ surrounded by a fictitious boundary $C$ (Fig. 2). Applying Green's second identity on $\vec{A}=\psi \hat{z}$ and $\vec{E}$ in region $R$ and after some manipulations yields

$$
\oint_{C}\left(j \omega \mu \psi \vec{J}_{S}+\vec{M}_{S} \times \nabla \psi\right) \cdot \hat{z} d C=0
$$

where

$$
\begin{aligned}
\vec{J}_{S} & =\hat{n} \times \vec{H}_{t} \\
\vec{M}_{S} & =\vec{E}_{t} \times \hat{n} .
\end{aligned}
$$

The solution of the two-dimensional homogeneous wave equation is, in general, a linear combination of modes. This implies that relationship (1) should be valid simultaneously for all the wave-equation solutions. Therefore, we have a set of $N$ integral equations. Expanding the equivalent currents on the boundary as a linear combination of orthogonal functions

$$
\begin{gathered}
J_{S}=\sum_{J=1}^{N} J_{j} j_{j} \\
M_{S}=\sum_{J=1}^{N} M_{j} m_{j} .
\end{gathered}
$$

The integral operator can be transformed into a matrix one as follows:

$$
[H] \cdot[J]^{C}=[U] \cdot[M]^{C}
$$

where the superscript $c$ denotes the column vector. The elements of matrices $[H]$ and $[\mathrm{U}]$ are

$$
\begin{aligned}
& h_{i j}=j \omega \mu \oint_{C} \psi_{i} j_{j} d C \\
& u_{i j}=-\oint_{C}\left(m_{j} \times \nabla \psi_{i}\right) \cdot \hat{z} d C
\end{aligned}
$$

and, subsequently,

$$
[J]^{C}=[Y] \cdot[M]^{C}
$$

being

$$
[Y]=[H]^{-1} \cdot[U] .
$$

Specifically, the solution modes of the wave equation in the guide are

$$
\psi_{m}=\sin \left[\frac{m \pi}{2 a}(y+a)\right] e^{ \pm j k_{x} x}
$$

where

$$
\left(\frac{m \pi}{2 a}\right)^{2}+k_{x}^{2}=k^{2}, \quad m=1,2, \cdots, M
$$

The \pm sign in the exponent indicates that both directions of propagation in the guide must be considered.

The electric and magnetic equivalent currents are expanded in a series of orthogonal functions. Considering port 1, we get

$$
\begin{aligned}
& j \omega \mu \int_{-a}^{a} \sin \left[\frac{m \pi}{2 a}(y+a)\right] e^{ \pm j k_{x} b} J_{1 z}(y) d y \\
& =-\left( \pm j k_{x}\right) e^{ \pm j k_{x} b} \int_{-a}^{a} \sin \left[\frac{m \pi}{2 a}(y+a)\right] M_{1 z}(y) d y
\end{aligned}
$$

being

$$
J_{1 z}=M_{1 z}=\sin \left[\frac{m \pi}{2 a}(y+a)\right]
$$

and whose solution is obviously immediate. The operator can be expressed in matrix form as

$$
\left[U_{1}\right] \cdot\left[J_{1}\right]^{C}=\left[H_{1}\right] \cdot\left[M_{1}\right]^{C} .
$$

In port 2 , we have

$$
\begin{gathered}
j \omega \mu \int_{-(\pi / 2)}^{\pi / 2} \sin \left[\frac{m \pi}{2 a}(y+a)\right] e^{ \pm j k_{x} x} J_{2 z}(\phi) d \phi \\
=\int_{-(\pi / 2)}^{\pi / 2}\left\{\cos (\phi)\left( \pm j k_{x}\right) \sin \left[\frac{m \pi}{2 a}(y+a)\right]+\frac{m \pi}{2 a} \sin (\phi)\right. \\
\left.\cdot \cos \left[\frac{m \pi}{2 a}(y+a)\right]\right\} e^{ \pm j k_{x} x} M_{2 \phi}(\phi) a d \phi
\end{gathered}
$$

being

$$
\begin{aligned}
& x=a \cos (\phi) \\
& y=a \sin (\phi) .
\end{aligned}
$$

The expansion functions in port 2 are now

$$
\begin{gathered}
J_{2 z}=\cos \left(q\left(\phi-\frac{\pi}{2}\right)\right) \\
M_{2 z}=\sin \left(q\left(\phi-\frac{\pi}{2}\right)\right) .
\end{gathered}
$$

The solution of (16) is carried out numerically for every one of the $2 M$ wave functions. The matrix formulation is again

$$
\left[U_{2}\right] \cdot\left[J_{2}\right]^{C}=\left[H_{2}\right] \cdot\left[M_{2}\right]^{C} .
$$

As (15) and (20) should be valid simultaneously, it can be written that

$$
\left[Y_{I}\right]=\left[\begin{array}{l}
{\left[U_{1}\right]} \\
{\left[U_{2}\right]}
\end{array}\right]^{-1} \cdot\left[\begin{array}{l}
{\left[H_{1}\right]} \\
{\left[H_{2}\right]}
\end{array}\right]
$$




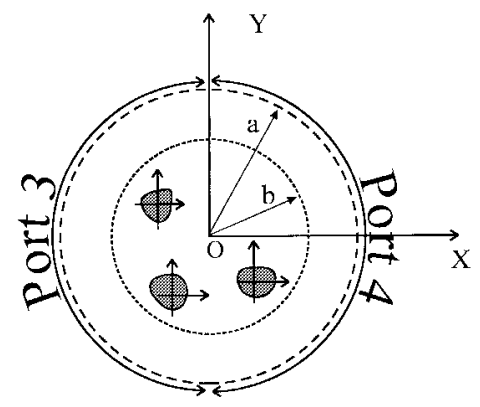

Fig. 3. Region surrounding the posts to be characterized analytically.

\section{ANALYTICAL Formulation FOR THE ADMITTANCE MATRIX OF REGION II}

Region II is depicted in Fig. 3. Two ports can be observed where the obstacle or group of obstacles is generally not centered. Outside the circumference surrounding the discontinuity, the wave function is

$$
\psi_{\mathrm{II}}=\sum_{n=-M}^{M}\left[a_{n} J_{n}(k \rho)+b_{n} H_{n}^{(2)}(k \rho)\right] e^{j n \phi}
$$

where the incident and reflected waves spectrum are related by a reflection matrix $\left[R_{c}\right]$ such that

$$
\left[b_{n}\right]^{C}=\left[R_{c}\right] \cdot\left[a_{n}\right]^{C} \text {. }
$$

Let us stress at this point that what suggests the analysis technique that is best suited for the scatterer characterization are its shape and electrical properties. A variety of techniques can then be introduced to compute $\left[R_{c}\right]$, ranging from modal analysis to numerical methods such as the unimoment method [11]. The introduction of such reflection matrix coefficients permits us a straightforward combination of different techniques under a single common procedure.

\section{A. Isolated Obstacle}

When the obstacle is centered, $\left[R_{c}\right]$ represents its reflection coefficient. However, when the obstacle is not centered, it is necessary to transform the incident and reflected waves in order to obtain a new reflection coefficient as follows:

$$
\left[R_{c}\right]^{\prime}=\left[T_{H}\right] \cdot\left[R_{c}\right] \cdot\left[T_{J}\right]
$$

This reflection coefficient is now valid outside a circumference centered in $O$ and containing the obstacle. The matrix $\left[T_{J}\right]$ expresses transformation of Bessel functions of the first kind centered in $O$, by means of Bessel functions of the first kind centered in $O^{\prime}$. The matrix $\left[T_{H}\right]$ provides the transformation of Hankel functions of the second kind centered in $O^{\prime}$ to their homologous ones centered in $O$. The transformation matrices can be readily obtained from Graf's addition theorem for Bessel functions [12]. A relationship between the electric and magnetic field can now be established in port 3 (Fig. 3) by

$$
H_{\phi}=-\frac{j \omega \varepsilon}{k^{2}} \frac{\partial E_{z}}{\partial \rho} .
$$

Expanding the electric field, as in (19), and introducing the inner product with regard to the magnetic field expanded in
(18), (25) can be formulated in matrix form as

$$
\left[Y_{33}\right]=\left[W_{3}\right] \cdot\left[Y_{\mathrm{II}}\right] \cdot\left[G_{3}\right]
$$

where

$$
\begin{aligned}
w_{q n} & =\frac{2}{\pi} \int_{\pi / 2}^{3 \pi / 2} h_{3 q}(\phi) e^{j n \phi} d \phi \\
g_{n l} & =\frac{1}{2 \pi} \int_{\pi / 2}^{3 \pi / 2} m_{3 l}(\phi) e^{-j n \phi} d \phi \\
{\left[Y_{\mathrm{II}}\right] } & =\frac{j}{\eta}\left(\left[J_{a}\right]+\left[H_{a}\right]\left[R_{c}\right]\right)^{-1} \cdot\left(\left[J_{a}\right]^{\prime}+\left[H_{a}\right]^{\prime}\left[R_{c}\right]\right)
\end{aligned}
$$

being

$$
\begin{aligned}
{\left[J_{a}\right] } & =\operatorname{diag}\left(J_{n}(k a)\right)_{n=-M}^{M} \\
{\left[J_{a}\right]^{\prime} } & =\operatorname{diag}\left(J_{n}^{\prime}(k a)\right)_{n=-M}^{M} \\
{\left[H_{a}\right] } & =\operatorname{diag}\left(H_{n}^{(2)}(k a)\right)_{n=-M}^{M} \\
{\left[H_{a}\right]^{\prime} } & =\operatorname{diag}\left(H_{n}^{(2)^{\prime}}(k a)\right)_{n=-M}^{M}
\end{aligned}
$$

Matrix $Y_{34}$ can be obtained in the following similar way:

$$
\left[Y_{34}\right]=\left[W_{3}\right] \cdot\left[Y_{\mathrm{II}}\right] \cdot\left[G_{4}\right]
$$

Finally, we are able to affirm that

$$
\begin{aligned}
& {\left[Y_{33}\right]=\left[Y_{44}\right]} \\
& {\left[Y_{34}\right]=\left[Y_{43}\right]}
\end{aligned}
$$

for those cases in which there exists symmetry.

\section{B. Multiple Posts}

When several objects are considered, the set can be characterized by a global reflection coefficient. In this way, the previous formulation for an isolated post can be used. In Fig. 3 there are $N$ scatterers of arbitrary cross section interacting among them. Each scatterer is characterized by a reflection coefficient $\left[R_{i}\right]$. Each pair of incident and reflected spectra $\left(\left[a_{i}\right],\left[b_{i}\right]\right)$ is referred to a local reference centered in the $i$ th scatterer, and then

$$
\left[b_{i}\right]^{C}=\left[R_{i}\right]\left[a_{i}\right]^{C}
$$

Noticing that the spectrum that impinges on the $i$ th scatterer includes the contribution of the source as well as the contribution of the fields scattered by the other posts, it can be written that

$$
\left[a_{i}\right]^{C}=\left[T_{0 i}\right] \cdot\left[I_{0}\right]^{C}+\sum_{\substack{m=1 \\ m \neq i}}^{M}\left[T_{m i}\right] \cdot\left[b_{m}\right]^{C}
$$

where $\left[I_{0}\right]^{c}$ is the source weights vector and $\left[T_{m i}\right]$ are the transformation matrices among the reference systems. Introducing (37) in (38) and rearranging terms,

$$
-\left[R_{i}\right] \cdot\left[T_{0 i}\right] \cdot\left[I_{0}\right]=-\left[b_{i}\right]+\left[R_{i}\right] \cdot \sum_{\substack{m=1 \\ m \neq i}}^{M}\left[T_{m i}\right] \cdot\left[b_{m}\right]^{C} .
$$

Forcing (39) on each one of the $M$ scatterers that constitute the discontinuity, a system of equations is obtained (being vectors 


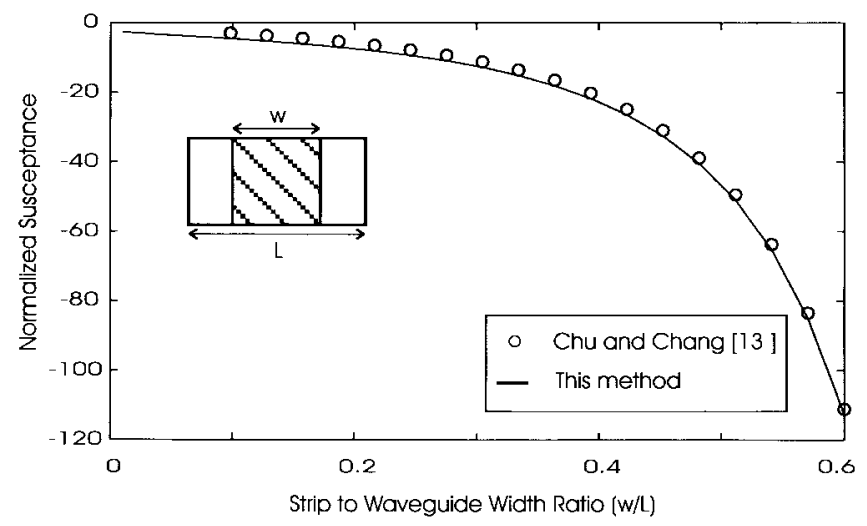

Fig. 4. Calculated normalized susceptance at $9 \mathrm{GHz}$ for a centered strip.

$\left[b_{i}\right]^{c}$, the unknown variables). Now referring all vectors to a common reference, a global reflection coefficient is obtained, as shown in (40), at the bottom of this page, where $[I]$ is the identity matrix.

It is worth stressing that this formulation envisages the mutual coupling among scatterers and, therefore, it is exact, provided that the scatterers individually taken have been correctly characterized.

Unlike other general-purpose methods, such as the finiteelement method, this technique permits us to isolate the contribution of every element involved in the problem. This feature turns out to be helpful when an iterative design process is in mind. It is clear that the admittance matrices characterizing homogeneous regions I and III should be computed only once and kept stored. Furthermore, if the design process involves a number of posts with fixed shapes and sizes, only the transformation matrices $\left[T_{i j}\right]$ should be recomputed on every step. Similarly, should the position of the posts remain fixed and their size and shape variable, only their matrix reflection coefficients would have to be recomputed.

\section{Results AND Discussion}

Once the admittance matrices have been obtained, they are connected, imposing continuity of the tangential components of the electric and magnetic field. The admittance matrix can be readily transformed into a generalized scattering matrix in order to study the influence of the posts in the reflection and transmission within the waveguide.

A few known examples have been chosen from the open literature to test validity of the method. First, Fig. 4 shows the normalized susceptance of a strip as a function of its width. Results compare well with those of Chu and Chang [13]. The

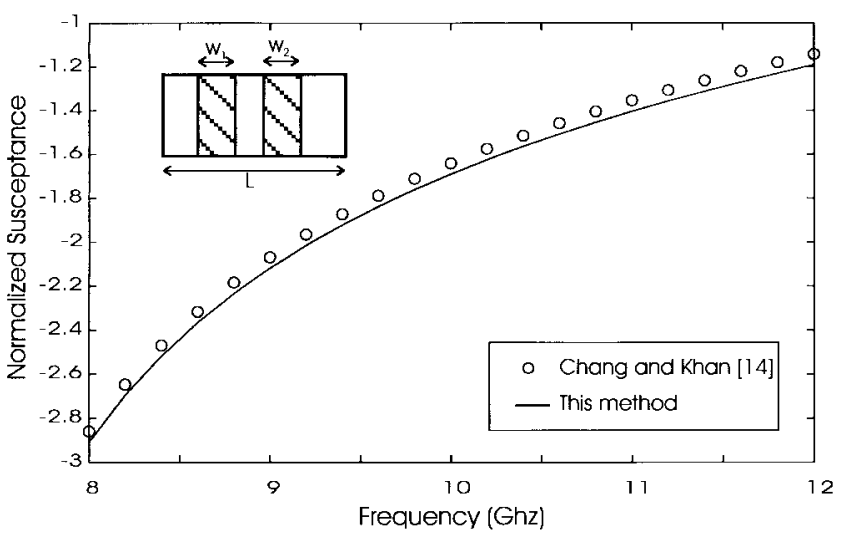

Fig. 5. Susceptance of a two-strip obstacle. $W_{1}=0.269 \mathrm{~cm}$, $W_{2}=0.274 \mathrm{~cm}, L=2.286 \mathrm{~cm}$.

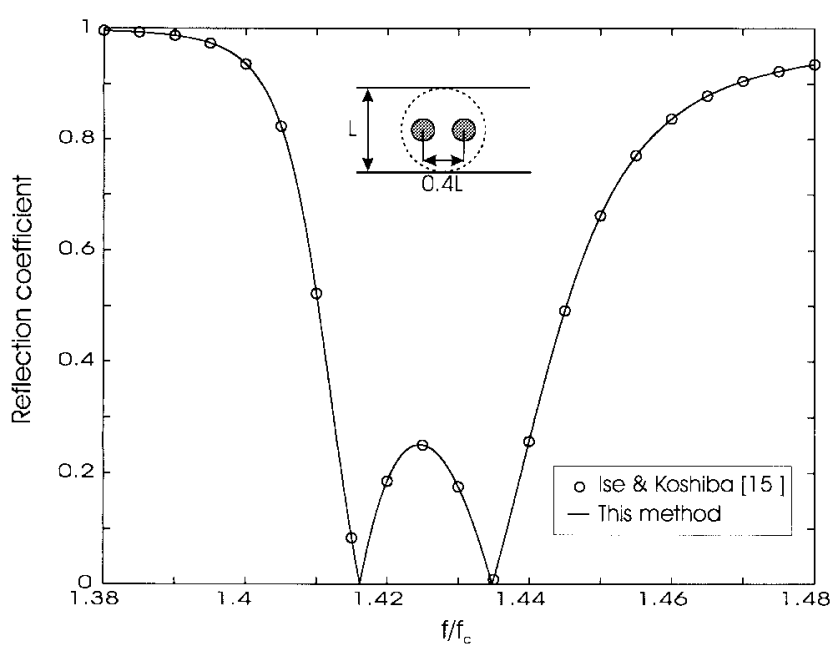

Fig. 6. Magnitude of reflection coefficient versus $f / f_{c}$ for the waveguide loaded with two centered posts of $r / L=0.05$ with $\varepsilon_{r}=112.5 . d / L=0.4$.

susceptance has been computed from the $S_{21}$ parameter as

$$
\bar{B}=\frac{B}{Y_{0}}=-\frac{2 \sin \phi_{21}}{\left|S_{21}\right|} .
$$

Fig. 5 now shows the susceptance of a configuration of two thin inductive perfectly conducting strips of unequal width $w_{1}$ and $w_{2}$. Results notably match those of Chang and Khan [14]. A completely different situation is solved in Figs. 6 and 7. $S$-parameters of two sets of circular cross-section dielectric posts are now shown. Results show good agreement with those obtained by Ise and Koshiba [15] and Hsu and Auda [6] using hybrid finite-element-method-boundary-element method and finite-element method, respectively.

$$
\left[R_{c}\right]=\left[\begin{array}{lllll}
{\left[T_{10}\right]} & \cdots & {\left[T_{i 0}\right]} & \cdots & {\left[T_{M 0}\right]}
\end{array}\right]\left[\begin{array}{ccccc}
{[I]} & \cdots & -\left[R_{1}\right]\left[T_{i 1}\right] & \cdots & -\left[R_{1}\right]\left[T_{M 1}\right] \\
\vdots & & \vdots & & \vdots \\
-\left[R_{i}\right]\left[T_{1 i}\right] & \cdots & {[I]} & \cdots & -\left[R_{i}\right]\left[T_{M i}\right] \\
\vdots & & \vdots & & \vdots \\
-\left[R_{M}\right]\left[T_{1 M}\right] & \cdots & -\left[R_{M}\right]\left[T_{i M}\right] & \cdots & {[I]}
\end{array}\right]^{-1} \cdot\left[\begin{array}{c}
{\left[R_{1}\right]\left[T_{01}\right]} \\
\vdots \\
{\left[R_{i}\right]\left[T_{0 i}\right]} \\
\vdots \\
{\left[R_{M}\right]\left[T_{0 M}\right]}
\end{array}\right]
$$




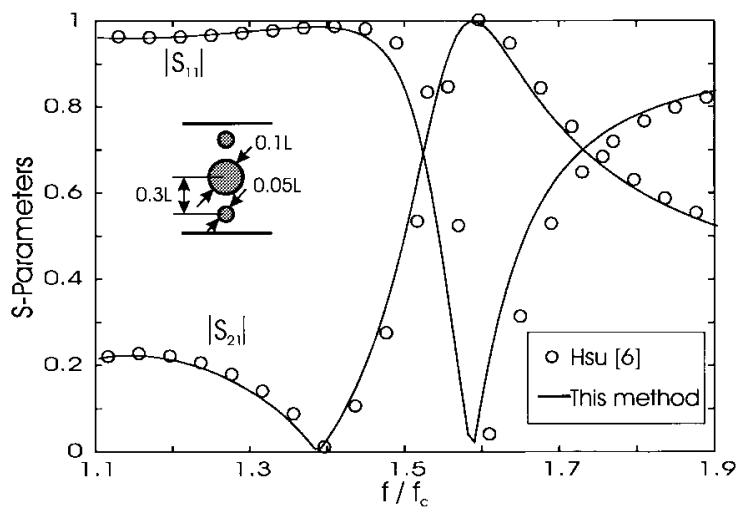

Fig. 7. $S$ parameters for a set of three cylindrical dielectric posts versus $f / f_{c}$.

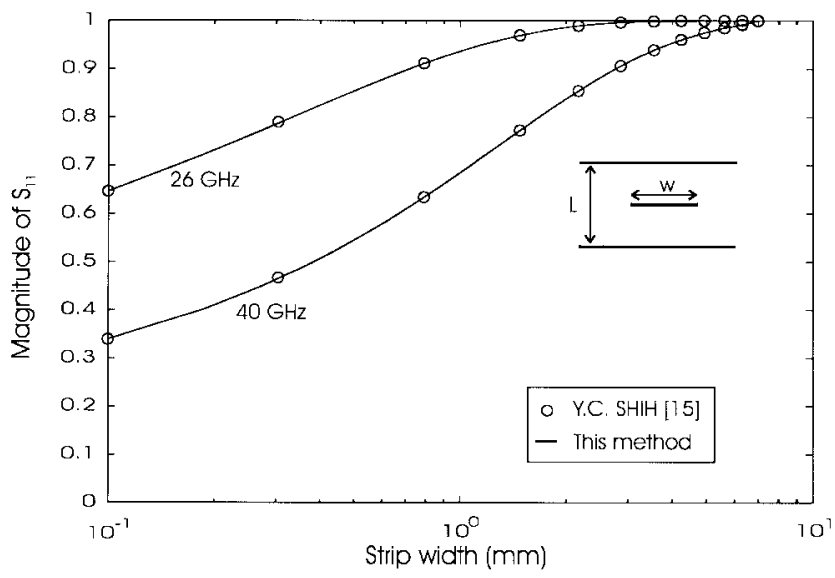

Fig. 8. Magnitude of reflection coefficient for axial strip of zero thickness.

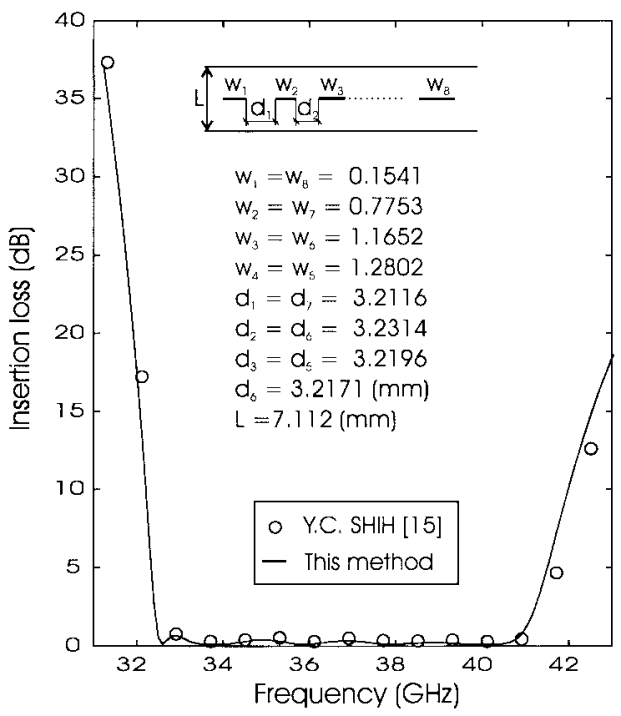

Fig. 9. An example of filter with relatively wide bandwidth.

Axial strips have been used to build microwave filters for years [16]. Figs. 8 and 9 permits us to compare our method with the well-known mode-matching method. The slight mismatch between the data in Fig. 9 is due to the nonzero thickness $(0.025 \mathrm{~mm})$ of the insert considered by Shih [16].
TABLE I

Admittance Matrix Computation Time in Seconds

\begin{tabular}{lccc}
\hline & $\begin{array}{l}\text { One } \\
\text { post }\end{array}$ & $\begin{array}{l}\text { Two } \\
\text { posts }\end{array}$ & $\begin{array}{c}\text { Three } \\
\text { posts }\end{array}$ \\
\hline Regions I \& III & $0.6 \mathrm{~s}$ & $0.6 \mathrm{~s}$ & $0.6 \mathrm{~s}$ \\
Region II & $0.4 \mathrm{~s}$ & $0.7 \mathrm{~s}$ & $1.6 \mathrm{~s}$ \\
\hline
\end{tabular}

A fundamental issue to be addressed is the computational cost. In Table I, computer resources required are shown. Computations have been performed on a $133-\mathrm{MHz}$ Pentium PC. As can be seen, the computation of the transition region admittance matrix is the most time-consuming task whenever one or two posts are considered. However, in a design process, this task would have to be accomplished only once. As a result, in practice, computation cost can be reduced significantly. Therefore, run time needed to compute $S$-parameters is much shorter than using the finite-elements method in a similar platform.

Another interesting point deals with the selection of expansion functions along the curvilinear ports. Although sinusoidal functions are known to be poorly convergent, only five modes have been needed to obtain the results shown. However, further refinement is under study to improve convergence.

\section{CONCLUSIONS}

A systematic and versatile method for the analysis of posts in waveguide has been proposed using network theory and the concept of generalized admittance. Although the method has been demonstrated only for dielectric rods and metallic strips, it is extensible to any other obstacle as long as it is correctly characterized by a reflection matrix coefficient. Therefore, this technique can be adapted to the characteristics of the obstacle, making possible the introduction of that numerical technique which best describes it. The method is fast and accurate, and could be a valuable tool to be included in real-time field solvers.

\section{REFERENCES}

[1] N. Marcuvitz, Waveguide Handbook. New York: McGraw-Hill, 1951.

[2] E. D. Nielsen, "Scattering by a cylindrical post of complex permittivity in a waveguide," IEEE Trans. Microwave Theory Tech., vol. MTT-17, pp. 148-153, Mar. 1969.

[3] J. C. Araneta, M. E. Brodwin, and G. A. Kriegsmann, "High-temperature microwave characterization of dielectric rods," IEEE Trans. Microwave Theory Tech., vol. MTT-32, pp. 1328-1335, Oct. 1984.

[4] Y. Leviatan and G. S. Sheaffer, "Analysis of inductive posts in rectangular waveguides," IEEE Trans. Microwave Theory Tech., vol. MTT-35, pp. 48-59, Jan. 1987.

[5] K. Ise and M. Koshiba, "Numerical analysis of $H$-plane waveguide functions by combination of finite and boundary elements," IEEE Trans. Microwave Theory Tech., vol. 36, pp. 1343-1351, Sept. 1988.

[6] C. G. Hsu and H. A. Auda, "Multiple dielectric posts in a rectangular waveguide," IEEE Trans. Microwave Theory Tech., vol. MTT-34, pp. 883-891, Aug. 1986.

[7] K. L. Wu, G. Y. Delisle, D. G. Fang, and M. Lecours, "Waveguide discontinuity analysis with a coupled finite-boundary element method," IEEE Trans. Microwave Theory Tech., vol. 37, pp. 993-998, June 1989.

[8] J. Abdulnour and L. Marchildon, "Boundary elements and analytic expansions applied to $H$-plane waveguide functions," IEEE Trans. Microwave Theory Tech., vol. 42, pp. 1038-1045, June 1994.

[9] F. Alessandri, G. Bartolucci, and R. Sorrentino, "Admittance matrix formulation of waveguide discontinuity problems: Computer-aided design 
of branch guide directional couplers," IEEE Trans. Microwave Theory Tech., vol. 36, pp. 364-403, Feb. 1988.

[10] N. Kishi and T. Okoshi, "Vectorial wave analysis of uniform-core optical fibers using a novel boundary integral method," IEEE Trans. Microwave Theory Tech., vol. 37, pp. 526-533, Mar. 1989.

[11] K. K. Mei, "Unimoment method for electromagnetic wave scattering," J. Electromag. Waves Applicat., vol. 1, no. 3, pp. 201-202, 1987.

[12] M. Abramovitz and I. A. Stegun, Eds., Handbook of Mathematical Functions (Applied Mathematics Series 55). Washington, DC: Nat. Bureau Stand., 1964.

[13] B. H. Chu and K. Chang, "Analysis of wide transverse inductive metal strips in a rectangular waveguide," IEEE Trans. Microwave Theory Tech., vol. 37, pp. 1138-1141, July 1989.

[14] K. Chang and P. J. Khan, "Coupling between narrow transverse inductive strips in waveguide," IEEE Trans. Microwave Theory Tech., vol. MTT-24, pp. 101-105, Feb. 1976.

[15] K. Ise and M. Koshiba, "Equivalent circuits for dielectric posts in rectangular waveguide," IEEE Trans. Microwave Theory Tech., vol. 37, pp. 1823-1825, Nov. 1989.

[16] Y. C. Shih, "Design of waveguide $E$-plane filters with all-metal inserts," IEEE Trans. Microwave Theory Tech., vol. MTT-32, pp. 695-704, July 1984.

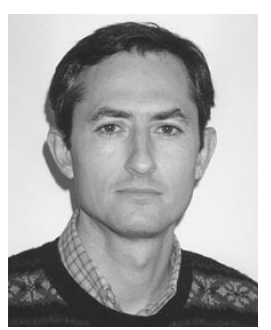

Alejandro Valero (S'92-M'97) was born in Madrid, Spain, on July 19, 1965. He received the M.S. degree in electrical engineering from Universidad Politecnica de Madrid, Madrid, Spain, in 1991, and the Ph.D. degree in electrical engineering from Universidad Politecnica de Valencia, Valencia, Spain, in 1997.

In 1992, he joined the Departamento de Comunicaciones, Universidad Politecnica de Valencia, where he is currently a Senior Lecturer. In 1999, he was on leave at the ElectroScience Laboratory, Ohio State University, Columbus, where he was involved in fast solution methods in electromagnetics and conformal antenna arrays. His current research interests includes fast numerical methods and asymptotic techniques for printed antennas and arrays.
Miguel Ferrando (S'81-M'83) was born in Alcoy, Spain, in 1954. He received the M.S.E. and Ph.D. degrees in telecommunication engineering from the Polytechnic University of Catalonia, Barcelona, Spain, in 1977 and 1982, respectively.

From 1977 to 1982, he was a Teaching Assistant in the Antennas, Microwave and Radar Group, Polytechnic University of Catalonia, and in 1982, became an Associate Professor. In 1990, he joined the Universidad Politécnica de Valencia, Valencia, Spain, where he is currently ViceChancellor and Professor of antennas and satellite communications in the Telecommunication Engineering College. He is currently involved in research activities in numerical methods, radar cross section, antennas, propagation, and telecommunication systems. 